\title{
Internet of things and multi-class deep feature-fusion based classification of tomato leaf disease
}

\author{
Rina Mahakud ${ }^{1}$, Binod Kumar Pattanayak ${ }^{1}$, Bibudhendu Pati ${ }^{2}$ \\ 'Department of Computer Science and Engineering, Siksha 'O' Anusandhan Deemed to be University \\ Institute of Technical Education and Research, Bhubaneswar, India \\ ${ }^{2}$ Department of Computer Science, Rama Devi Women’s University, Bhubaneswar, India
}

\begin{tabular}{l} 
Article Info \\
\hline Article history: \\
Received Sep 17, 2021 \\
Revised Dec 20, 2021 \\
Accepted Dec 24, 2021 \\
\hline
\end{tabular}

Keywords:

AlexNet

CNNs

SVM

Tomato leaf disease

identification

Transfer learning

\begin{abstract}
A deep transfer learning (deep-TL) classification model has been proposed to diagnose tomato leaf disease. The main challenge of inaccurate classification of a convolution neural network (CNN) model was the availability of the small-sized dataset. This model deals with the challenges like availability of small-sized and imbalanced datasets. The proposed Alex support vector machine (SVM) fused hybrid classification (ASFHC) model is based on fully fusion technology that avoids overfitting to classify the type of disease in tomato leaves. The proposed model achieves the best performance in terms of accuracy by data augmentation of the training data. It uses a pre-trained network for feature extraction with the modification of architecture by concatenating two layers FC6 and FC7 (fully connected layer), plus a linear SVM classifier for classification of the disease. The uniqueness of the research is although the dataset is not balanced, the performance of the model has achieved the maximum. Compared with VGG 16 and VGG 19, the proposed model (ASFHC) has been evaluated using different measuring parameters, indicating remarkable computation time for implementation in the internet of things (IoT) domain. The overall accuracy attained by the model is $99.62 \%$.
\end{abstract}

This is an open access article under the CC BY-SA license.

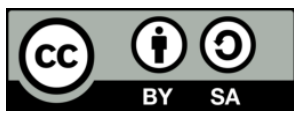

\section{Corresponding Author:}

Rina Mahakud

Department of Computer Science and Engineering, Siksha 'O' Anusandhan Deemed to be University

Institute of Technical Education and Research

Bhubaneswar, India

Email: rinamahakud@gmail.com

\section{INTRODUCTION}

Tomato is a useful vegetable that is rich in vitamin $\mathrm{C}$, potassium, folate, and vitamin $\mathrm{K}$. This plant has some medicinal values. It is advisable to consume in order to reduce the risk of heart disease and cancer. In 2020, world processing tomato council (WPTC) world estimate of tomato production is around 39.2 million metric tons, mainly due to the increase of the Chinese forecast to 5.6 million tones [1]. The growth cycle of the tomato plant is relatively short, that is about three to five months with an average temperature 10 to 20 degrees [2]. It is very difficult for a farmer to identify the diseases in tomato plants from their past experience because of climate change. In the manual case of cultivation, the process is laborintensive, cost-effective and time-consuming. So, they need domain expert visit to the field, which is costly. It is challenging to protect healthy plants from diseases to provide quality and quantitative products in the market. Therefore, if diseases are appropriately diagnosed, then precautions can be taken. To replace the expert's advice in traditional method of cultivation, it is very much of necessary to automate the disease identification process at very early stage [3]. The type of leaf disease classification by visual observation is 
prone to error due to environmental stimuli such as shadows, reflections of sunshine, fog, and high humidity [4]. Recently advancement in computational tools such as image processing, remote sensing, artificial intelligence and deep learning helped to achieve successful application. In last 10 years, deep learning (DL) had been applied in various fields like video classification, object detection, and human action recognition. Freshly DL has also been successfully applied in agricultural sector for disease identification and prediction of disease [5], [6]. In the past decade, the classification algorithm such as support vector machine (SVM) [7], k-nearest neighbor (KNN) [8], artificial neural network (ANN) [9], particle swarm optimization [10], random forest (RF) [11] and so on were used for plant disease identification. SVM is a popular supervised machine learning classifier used for solving both binary and multi class classification problem. Thus, pre-trained convolution neural network (CNN) network with SVM classifier improves the performance of the model.

The main work of this paper is revolved around the vegetable tomato. This is a vegetable which can be cultivated throughout the year. The climate changing factors that are humidity, heavy rain, global radiation, and temperature. That can affect the growth of the crop [12] and due to these factors' plants got affected by the diseases. So, there are a lot of research work undertaken by the researcher for leaf diseases identification of the tomato plant using various models. A convolution neural network (CNN) model was proposed in [13] for the tomato plant's leaf-based classification. In this CNN Model, 1400 leaf images had been trained in suggested 8 layers and $98.4 \%$ of accuracy was obtained. Verma et al. [14] developed a CNN architectures-based model which works in two different modes for extracting the features and then the extracted feature set was trained in a multiclass SVM to get the final output. The author compared the accuracy of the proposed AlexNet model with other two networks SqueezeNet and Inception V3. The AlexNet resulting the highest accuracy in transfer learning and feature extraction approaches $89.69 \%$ and 93.4\%, respectively for tomato late blight disease. Ashqar and Abu-Naser [15] presented a full-color neural network model to extract features in two sub sections. The first section of the model is feature extraction which consists of 4 layers of convolutional layers, rectified linear unit (ReLu) and max pooling layer. The second section of the model is flattened layer by using two dense layers for both approaches. Luna et al. [16] developed a pre-trained deep learning AlexNet architecture to identify the three tomato leaf diseases. The author utilizes the model AlexNet both anomaly detection and disease detection. The proposed model had an accuracy of 91.6 percent based on 36 samples. Ferentinos [17] developed an optimal deep learning model and used dataset contains 87,848 infected images and healthy leaves out of 25 plant species from open datasets. The author concluded that the best performance given by VGG convolutional neural network with $99.53 \%$ of success rate in the classification in comparison to AlexNet one weird trick batch (AlexNetOWTBn) with 99.49\% of success rate. Hassanien et al. [18] proposed a mathematical model moth-flame optimization (MFO) and rough set (MFORSFS) approach to automatically detect powdery mildew and early blight of tomato disease. Textural pattern features are extracted by using Gabor transform of diseased tomato leaves. Here author represented a comparison between particle swarm optimization and genetic algorithms with rough sets from which rough set provide better results. MFORS-based classification offers a $91.5 \%$ result as compared to maximum relevance minimum redundancy (mRMR) based method.

A Transfer learning approach is proposed in this present work to identify and classify the type of disease in real time. For implementation of the model a pretrained network is used with the modification of architecture. The prosed fused hybrid model is a classification model based on deep CNN model. The main contributions of this work are follows:

- A fused hybrid model is proposed, which can provide better accuracy with faster execution time withan imbalanced dataset by using field images as well as standard dataset to achieve a useful detection of tomato plant disease.

- A Pre-trained transfer learning architecture based on fusion concept is used to analyze tomato leaf disease characteristics, with the modification of concatenating two layers FC6 and FC7 (fully connected layer).

\section{MATERIALS AND METHOD}

The classification of the disease identification in tomato plant is shown in Figure 1. In this section, detail of image dataset creation from internet of things (IoT) module and implementation of suggested classification of the disease models are elaborated. The proposed model is broadly divided into three units such as data acquisition (IoT modules sensed the image data), processing unit (using CNN) and classification unit are described in subsequent subsection.

\subsection{Image dataset}

Sensors are placed in the field to capture images at regular intervals during crop growth in order to monitor for infection. This proposed model is comprised of a device known as the Rasberry PI, which is a quad-core 64-bit advanced reduced instruction set computing (RISC) machines (ARM) processor running at 
1.2 GHz. The web camera is linked to the processor device. Here, a digital camera, web camera, or closedcircuit television (CCTV) can be linked to the processor to continuously capture images of leaves and generate a database of the leaves. All the modules are connected through IoT network. But in the proposed IoT based disease identification scheme, the dataset is collected from 'Plant village' database. The dataset contains a total of 6660 images, out of which 6578 images are diseased tomato leaf images caused by yellow leaf curl virus and mosaic virus, and 82 of which are healthy tomato leaf images. All the images are preprocessed for resized to $227 \times 227 \times 3$. The dataset contains tomato leaf images which are grouped into three classes as tomato mosaic virus, tomato yellow leaf curl, and tomato healthy is shown in Figure 2 . These three classes are shown in Figures 2(a)-(c) respectively. We had taken $80 \%$ and $20 \%$ of the samples for training and testing purpose respectively. The samples are chosen by the model randomly for each execution.

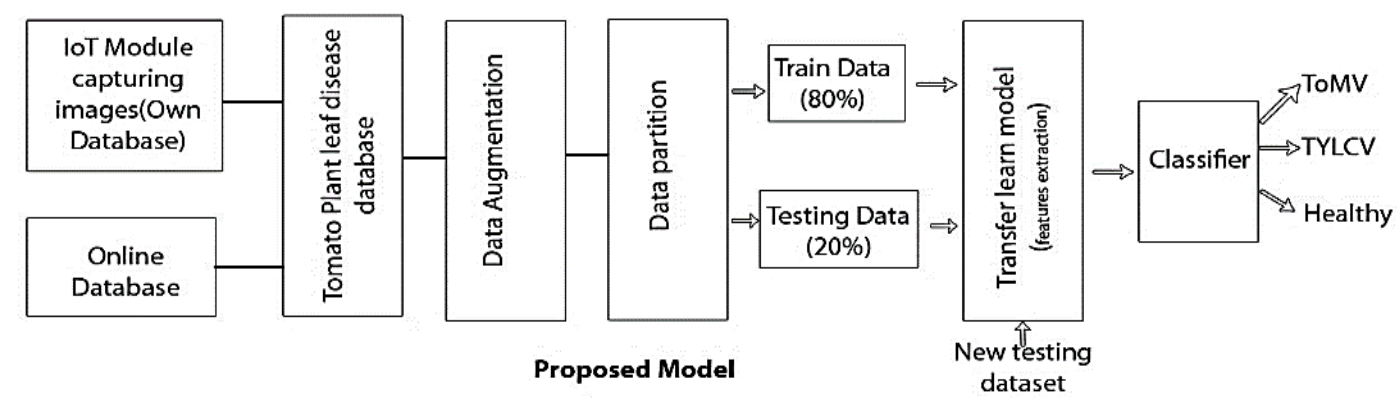

Figure 1. Block diagram of proposed model for identification of tomato leaf diseases

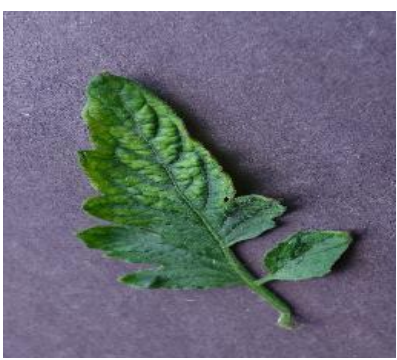

(a)

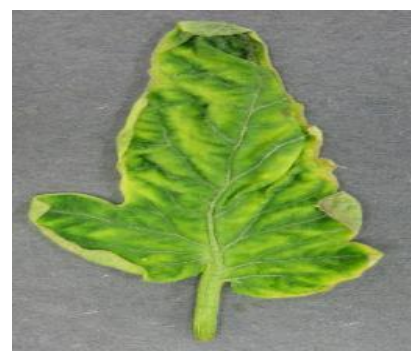

(b)

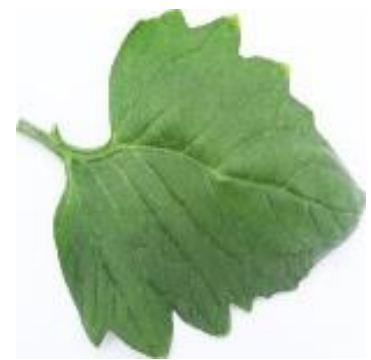

(c)

Figure 2. Sample images of tomato leaf: (a) tomato mosaic virus, (b) tomato yellow leaf curl, and (c) tomato healthy

\subsection{Data augmentation}

Data augmentation is a manipulating technique which is done by simple rotation of images (i.e., 90 degrees right and left rotation and 180 degrees rotation) and flipping operation (i.e., vertical flipping and horizontal flipping) to overcome the problem of overfitting, we applied data augmentation. By augmentation, the number of sample images are increases to six times to the number of images to the applied augmentation images. As a result, the chance to learn the appropriate feature is increased for the network [19]. Table 1 presents the detail of each class of tomato leaf disease used during the experimentation.

Table 1. Details of image dataset for tomato leaf disease used in the study

\begin{tabular}{lcccc}
\hline \multicolumn{1}{c}{ Leaf diseases } & $\begin{array}{c}\text { Number of images } \\
\text { (original) }\end{array}$ & $\begin{array}{c}\text { Number of images used } \\
\text { for (augmentation) }\end{array}$ & $\begin{array}{c}\text { Number of images } \\
\text { used for (Training) }\end{array}$ & $\begin{array}{c}\text { Number of images } \\
\text { used for (Testing) }\end{array}$ \\
\hline $\begin{array}{l}\text { Tomato mosaic virus } \\
\text { (ToMV) }\end{array}$ & 1110 & 910 & 5460 & 200 \\
$\begin{array}{l}\text { Tomato yellow leaf curl } \\
\text { virus (TYLCV) }\end{array}$ & 5468 & 5268 & 31608 & 200 \\
Total & 6578 & 6178 & 37068 & 400 \\
\hline
\end{tabular}




\section{METHOD}

Generally, transfer learning is the approach of deep learning, which is used for deep feature extraction. In this model, we include three pre-trained model for feature extraction. In this work, the feature extraction is done through concatenating two fully connected layers (FCL) and from the fully connected layers of (FC6 and FC7), extracted features are feed to the linear SVM classifier for classify the disease during training the model.

Process involves in the feature extraction the deep learning approach applied in this model, involves in two process that are i) feature extraction and ii) classification. Feature extraction processes can be carried out with several convolution layers added with max-pooling layers and output of convolution layers are connected to ReLu [20] as a non-linear activation function. The convolution layer involves in the process can be learned in supervised or unsupervised manner contains a set of filters. It applies the convolution operation on the input image, then the result will be applied to the next layer. In convolution layer, it is calculated by using the formula as given in (1).

$$
x=\frac{n+2 p-d}{s}+1
$$

Where, $n=$ image size having height and width, $\mathrm{p}$ is zero padding, $\mathrm{d}$ is the filter size and $\mathrm{s}$ is the size of the stride.

The pooling layer is used to reduce the dimensions of the feature map due to which it summarizes the features generated by convolution layers to makes the model more robust. Generally, two types of pooling are taken in to the consideration that are average and max pooling. In case of average polling, the average elements are calculated within the receptive field to send the output array. But in max pooling maximum number of elements selected from the region in the feature map. Thus, max pooling provides most prominent features in the region.

In this work, we propose transfer learning approach to extract the deep features that are the abstract features. From the related case studies of a methodology based on deep learning Ding et al. [19] focused on the feature extraction and classification. During experimentation, it was found that the fully connected layer FC6 is providing higher accuracy in classification than FC7. Sethy et al. [21], described an in-depth featurebased leaf disease identification model. From there experimental study, the authors concluded that FC6 and FC7 give better result as compared to FC8. In the proposed model, we have taken those deep features and do the concatenation (i.e., concatenation for FC6 and FC7). So, here in this work the deep features extracted from FC6 and FC7is considered. From FC6 4096 number of features are extracted and similarly from FC7 also 4096 number of features are extracted. Then the extracted features from FC6 and FC7 are fused together to enhance the dimension of features. The features fusion process is done by concatenation the two features i.e, FC6 and FC7 to form a high dimensional feature. Here the dimension of the features is added to provide $4096+4096=8192$. With consideration of this number of features the liner SVM classifying three varieties of tomato disease such as tomato mosaic virus (ToMV), tomato yellow leaf curl virus (TYLCV), and tomato healthy.

Said et al. [22] suggested for image classification in machine learning most preferable classifier is SVM. This classifier separates the datapoint using a line of hyperplane for dataset labelling during the training process for tomato leaf disease. In this paper linear-SVM is used for disease classification based on deep features of pre-trained model Alexnet. "To train the SVM, the function 'fit class error-correcting output codes (fitcecoc)' was used. This function returns full trained multiclass error-correcting output of the model. The function 'fitcecoc' uses $\mathrm{K}(\mathrm{K}-1) / 2$, binary SVM model, using one-vs-all coding design. Here, $\mathrm{K}$ is a unique class label. Because of error correcting output codes and one-vs-all coding design of SVM, the performance of classification models was enhanced [21]." The proposed model involved the following steps are given in Algorithm 1.

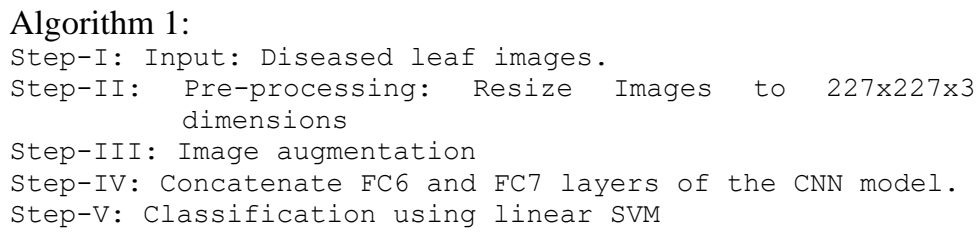

\section{RESULTS AND DISCUSSION}

In this research model, CNN based transfer learning is used to identify tomato leaf disease with giving important to the efficiency of the model in classification and execution time taken by the model. 
MATLAB 2020 a with add on as deep leaning is used for experimental study with the system having, configuration Intel i7 $9^{\text {th }}$ generation processor, 16 GB DDR4 RAM, 250 GB SSD \& 4TB HDD, NVIDA 1050Ti 4GB DDR5 GPU with NVIDA cuda_11.1.0_456.43_win10. The classification model's performance is evaluated through accuracy. To get maximum accuracy several iterations to be performed and some training time will be required for the performance. In this transfer learning approach, the experiment is carried out in two phases i.e. training phase and testing phase.

From subsection 3.1, it was noticed that proposed model performs well in the FC6 and FC7 layer for classification. From this observation we included those deep features and concatenate these fully connected layers to perform better results. In this section, we experimented a transfer learning pre-trained models based on CNN. The hyper parameters used in transfer learning methods are stochastic gradient descent (SGD), 0.001 for the initial learning rate, 50 for the number of epochs, 0.9 for momentum, 0.2 for drop out, and 16 for the minibatch scale. The minibatch size can be $8,16,32,64,128$ or 256 , depending on the GPU's ability. The simulated result of proposed (ASFHC) model, VGG 16, VGG 19 classification model's is represented by confusion matrix given in Figure 3. Figure 3(a) shows the better classification result in camparision to Figures 3(b) and (c).

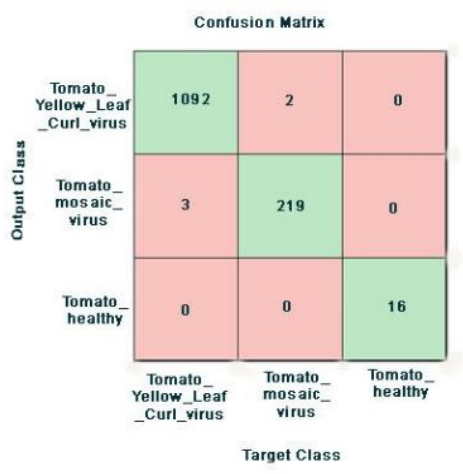

(a)

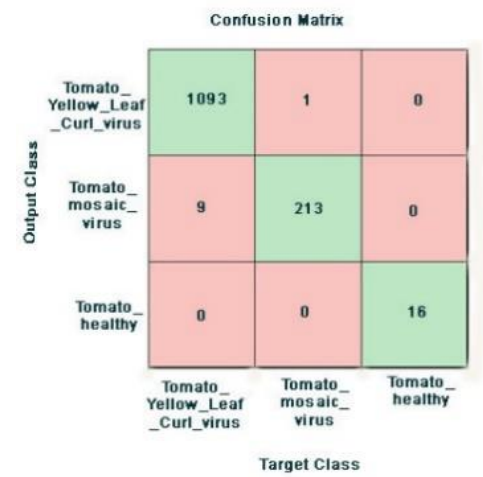

(b)

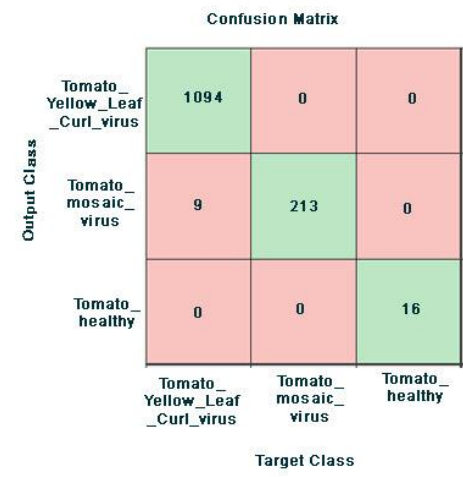

(c)

Figure 3. Confusion matrix for the (a) ASFHC, (b) VGG 16, and (c) VGG 19

In this above confusion matrix, each rows represent the actual class and each column represent the prediction class whereas diagonal values represent each leaf classes correctly calculated shown in green color other values shown in pink color misclassified. In the proposed ASFHC model, first row the confusion matrix contains total 1094 instants out of which 1092 instants correctly classified as TYLCV and only 2 instants were wrongly classified as ToMV is shown as error. In second row 219 correctly classified as ToMV and 3 instants were misclassified as TYLCV. In third row all 16 instants are classified as healthy. Thus, the performance measures of tomato disease plant are given in Table 2.

Table 2. Measurement parameters of CNN models with deep features

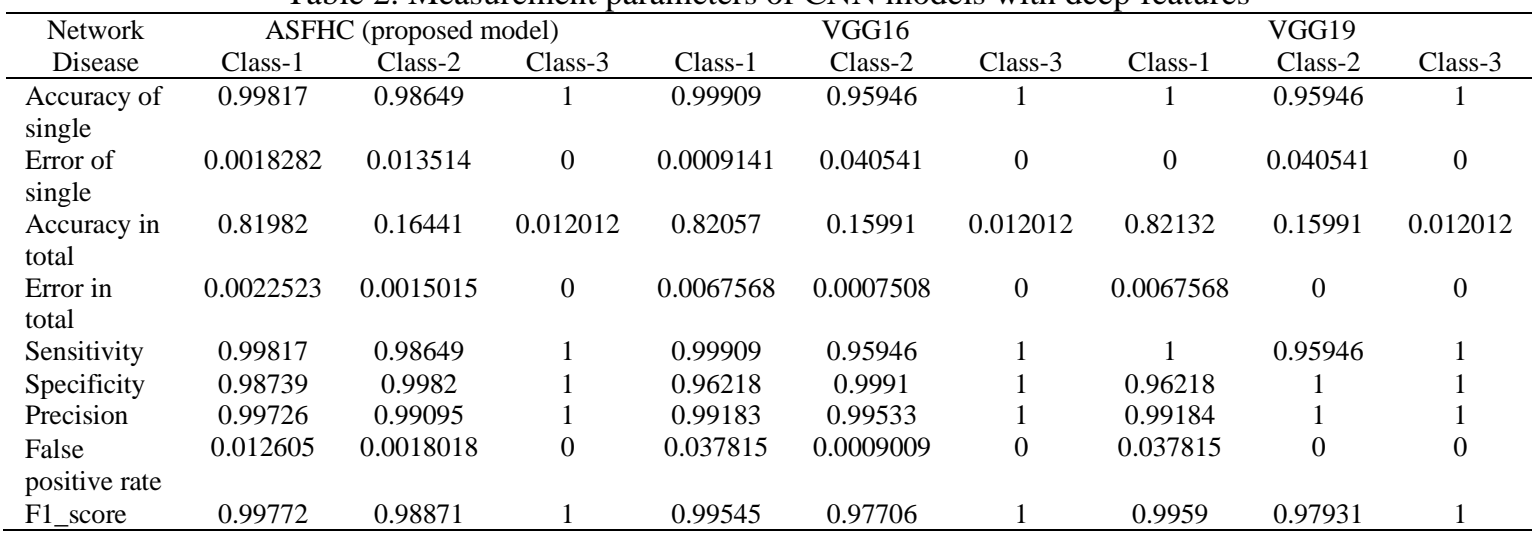

*Tomato_mosaic_virus=> class1, Tomato_Yellow_Leaf_Curl_Virus=>class-2, Tomato_healthy=>class-3 
From Table 2 the performance measuring parameters of each disease including healthy tomato plant was indicated. The proposed approach not only identified the disease but also distinguishes the specific types of diseases. The proposed model provides overall performance is given in Table 3.

Table 3. Overall performance of the proposed model

\begin{tabular}{lcccccccc}
\hline \multicolumn{1}{c}{ Network } & Accuracy & Error & Sensitivity & Specificity & Precision & FPR & F1_score $\begin{array}{c}\text { Execution } \\
\text { time }(\mathrm{sec})\end{array}$ \\
\hline ASFHC (proposed model) & $\mathbf{0 . 9 9 6 2}$ & $\mathbf{0 . 0 0 3 8}$ & $\mathbf{0 . 9 9 4 9}$ & $\mathbf{0 . 9 9 5 2}$ & $\mathbf{0 . 9 9 6 1}$ & $\mathbf{0 . 0 0 4 8}$ & $\mathbf{0 . 9 9 5 5}$ & $\mathbf{4 6}$ \\
VGG16+ Linear SVM & 0.9925 & 0.0075 & 0.9862 & 0.9871 & 0.9957 & 0.0129 & 0.9908 & 175 \\
VGG19+ Linear SVM & 0.9932 & 0.0068 & 0.9865 & 0.9874 & 0.9973 & 0.0126 & 0.9917 & 237 \\
\hline
\end{tabular}

From Table 3, it was observed that by using fused features of FC6 and FC7, the ASFHC achieves highest accuracy which is indicates in bold. All the measuring parameters are obtained the maximum value. But as this model is implemented in IoT domain, the response of the model should be faster. On the basis of execution time, it was observed that ASFHC model provides the result in just 46sec. whereas VGG 16 takes $175 \mathrm{sec}$ and VGG 19 takes 237sec. Hence, the ASFHC model with linear SVM is the best classifier model can be considered in IoT domain. The proposed model compares the accuracy with existing model are given in Table 4.

From the Table 4 it can be summarized that the plant village dataset was used by most of the authors. It contains thousands of tomato leaf images. Most of the above research approach includes SVM classifier to detect the diseases. But only Sethy et al. [21] able to provide computation time as $48.65 \mathrm{sec}$. The proposed model i.e., Alex SVM fused hybrid classification (ASFHC) with fused features (FC6 \& FC7) and linear SVM is the chosen classifier to classify and identify the tomato leaf disease with an accuracy of 99.62\% in computation time $46 \mathrm{sec}$ which can be implemented IoT application domain. In this proposed model may have limitations, that it will provides more accurate result in color images rather than gray scale images because this model implemented in IoT domain which collect the real time images at normal environmental condition.

Table 4. Comparison of various methods with proposed model

\begin{tabular}{|c|c|c|c|c|}
\hline Authors & Dataset & Methodology & Accuracy & Computation time (Sec) \\
\hline Verma et al. [14] & Plant village dataset & $\begin{array}{l}\text { CNN architecture (i.e., } \\
\text { transfer learning and } \\
\text { feature extraction) } \\
+ \text { multiclass SVM }\end{array}$ & $93.4 \%$ & 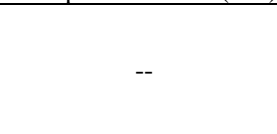 \\
\hline Sethy et al. [21] & $\begin{array}{l}\text { http://bcch.ahnw.gov.cn/Right. } \\
\text { aspx }\end{array}$ & Deep Learning + SVM & $97.62 \%$ & 48.65 \\
\hline Brahimi et al. [23] & Plant village dataset & $\begin{array}{l}\text { Pre-processing }+ \text { feature } \\
\text { extraction }+S V M \text { or } \\
\text { Random Forest }\end{array}$ & $98.660 \%$ & $\begin{array}{l}\text { Not Specified but want } \\
\text { to reduce computation } \\
\text { time }\end{array}$ \\
\hline Durmus et al. [24] & Plant village dataset & $\begin{array}{l}\text { Comparative study of } \\
\text { AlexNet and } \\
\text { SqueezeNet }\end{array}$ & $95.65 \%$ & 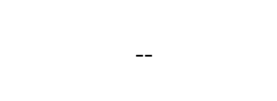 \\
\hline Hong et al. [25] & Plant village dataset & Deep CNN model & $97.10 \%$ & -- \\
\hline Altuntas et al. [26] & Plant village dataset & Deep CNN model & $96.99 \%$ & \\
\hline ASFHC (Proposed Model) & Plant village dataset & $\begin{array}{l}\text { Deep CNN+linear } \\
\text { SVM }\end{array}$ & $99.62 \%$ & $\begin{array}{l}-- \\
46\end{array}$ \\
\hline
\end{tabular}

\section{CONCLUSION}

This proposed model focuses on deep-CNN transfer learning approach to identify tomato leaf disease with high accuracy of $99.62 \%$. In this current research, we evaluate the performance of the model with linear SVM. The main aim of this research work is to automate the disease identification as it is implemented in IoT domain. Here, we are avoiding overfitting by concatenating FC6 and FC7 of the architecture due to which all the features are getting concatenated to provide best execution time just at 46 sec. These network models are evaluated with other existing models out of which ASFHC model performs best execution time as compared to other models, which fits to be implemented in IoT domain. This research can be further carried forward with a comparative analysis with other existing methods with more variety of tomato diseases to achieve better performance. 


\section{REFERENCES}

[1] Tomatonews, WPTC Crop situation as of 3 July 2020, 2020. Accessed: Mar. 27, 2020. [Online]. Available: http://www.tomatonews.com/en/wptc-crop-situation-as-of-3-july-2020_2_1072.html

[2] T. R. Gadekallu et al., "A novel PCA-whale optimization-based deep neural network model for classification of tomato plant diseases using GPU," Journal of Real-Time Image Processing, vol. 18, pp. 1383-1396, 2021, doi:10.1007/s11554-020-00987-8.

[3] G. Geetha, S. Samundeswari, G. Saranya, K. Meenakshi, and M. Nithya, "Plant Leaf Disease Classification and Detection System Using Machine Learning," Journal of Physics: Conference Series, vol. 1712, 2020, doi: 10.1088/1742-6596/1712/1/012012.

[4] I. Ahmad, M. Hamid, S. Yousaf, S. T. Shah, and M. O. Ahmad, "Optimizing pretrained convolutional neural networks for tomato leaf disease detection," Complexit, 2020, doi: 10.1155/2020/8812019.

[5] P. Tm, A. Pranathi, K. SaiAshritha, N. B. Chittaragi, and S. G. Koolagudi, "Tomato Leaf Disease Detection Using Convolutional Neural Networks," 2018 Eleventh International Conference on Contemporary Computing (IC3), 2018, pp. 1-5, doi: 10.1109/IC3.2018.8530532.

[6] S. Verma, A. Chug, and A. P. Singh, "Prediction Models for Identification and Diagnosis of Tomato Plant Diseases," 2018 International Conference on Advances in Computing, Communications and Informatics (ICACCI), 2018, pp. 1557-1563, doi: 10.1109/ICACCI.2018.8554842.

[7] L. M. Griffel, D. Delparte, and J. Edwards, "Using Support Vector Machines classification to differentiate spectral signatures of potato plants infected with Potato Virus Y," Computers and Electronics in Agriculture, vol. 153, pp. 318-324, 2018, doi: 10.1016/j.compag.2018.08.02.

[8] D. K. Behera, M. Das, S. Swetanisha, and P. K. Sethy, "Hybrid model for movie recommendation system using content K-nearest neighbors and restricted boltzmann machine," Indonesian Journal of Electrical Engineering and Computer Science, vol. 23, no. 1, pp. 445-452, 2021, doi: 10.11591/ijeecs.v23.i1.pp445-452.

[9] K. Gangadharan, G. R. N. Kumari, D. Dhanasekaran, and K. Malathi, "Detection and classification of various pest attacks and infection on plants using RBPN with GA based PSO algorithm," Indonesian Journal of Electrical Engineering and Computer Science, vol. 20, no. 3, pp. 1278-1288, 2020, doi: 10.11591/ijeecs.v20.i3.pp1278-1288.

[10] S. Sengupta and A. K. Das, "Particle Swarm Optimization based incremental classifier design for rice disease prediction," Computers and Electronics in Agriculture, vol. 140, pp. 443-451, 2017, doi: 10.1016/j.compag.2017.06.024.

[11] J. Kodovsky, J. Fridrich, and V. Holub, "Ensemble Classifiers for Steganalysis of Digital Media," in IEEE Transactions on Information Forensics and Security, vol. 7, no. 2, pp. 432-444, April 2012, doi: 10.1109/TIFS.2011.2175919.

[12] K. Golhani, S. K. Balasundram, G. Vadamalai, and B. Pradhan, "A review of neural networks in plant disease detection using hyperspectral data," Information Processing in Agriculture, vol. 5, no. 3, pp. 354-371, 2018, doi: 10.1016/j.inpa.2018.05.002.

[13] M. Agarwal, S. Kr. Gupta, and K. K. Biswas, "Development of Efficient CNN model for Tomato crop disease identification," Sustainable Computing: Informatics and Systems, vol. 28, 2020, doi: 10.1016/j.suscom.2020.100407.

[14] S. Verma, A. Chug, and A. P. Singh, "Application of convolutional neural networks for evaluation of disease severity in tomato plant," Journal of Discrete Mathematical Sciences and Cryptography, vol. 23, no. 1, pp. 273-282, 2020, doi: 10.1080/09720529.2020.1721890.

[15] B. A. M. Ashqar and S. S. Abu-Naser, "Image-based tomato leaves diseases detection using deep learning," International Journal of Academic Engineering Research (IJAER), vol. 2, no. 12, pp. 10-16, 2018

[16] R. G. de Luna, E. P. Dadios, and A. A. Bandala, "Automated Image Capturing System for Deep Learning-based Tomato Plant Leaf Disease Detection and Recognition," TENCON 2018 - 2018 IEEE Region 10 Conference, 2018, pp. 1414-1419, doi: 10.1109/TENCON.2018.8650088

[17] K. P. Ferentinos, "Deep learning models for plant disease detection and diagnosis," Computers and Electronics in Agriculture, vol. 145, pp. 311-318, 2018, doi: 10.1016/j.compag.2018.01.009.

[18] A. E. Hassanien, T. Gaber, U. Mokhtar, and H. Hefny, "An improved moth flame optimization algorithm based on rough sets for tomato diseases detection," Computers and Electronics in Agriculture, vol. 136, pp. 86-96, 2017, doi: 10.1016/j.compag.2017.02.026.

[19] L. Ding, H. Li, C. Hu, W. Zhang, and S. Wang, "Alexnet feature extraction and multi-kernel learning for object-oriented classification," Int. Arch. Photogramm. Remote Sens. Spat. Inf. Sci, pp. 277-281, 2018, doi: 10.5194/isprs-archives-XLII-3-2772018

[20] A. A. Abbood, Q. M. Shallal, and M. A. Fadhel, "Automated brain tumor classification using various deep learning models: a comparative study," Indonesian Journal of Electrical Engineering and Computer Science, vol. 22, no. 1, pp. 252-259, 2021, doi: 10.11591/ijeecs.v22.i1.pp252-259.

[21] P. K. Sethy, N. K. Barpanda, A. K. Rath, and S. K. Behera, "Deep feature-based rice leaf disease identification using support vector machine," Computers and Electronics in Agriculture, vol. 175, 2020, doi: 10.1016/j.compag.2020.105527.

[22] N. S. M. Said, H. Madzin, S. K. Ali, and N. S. Beng, "Comparison of color-based feature extraction methods in banana leaf diseases classification using SVM and K-NN," Indonesian Journal of Electrical Engineering and Computer Science, vol. 24, no. 3, pp. 1523-1533, 2021, doi: 10.11591/ijeecs.v24.i3.pp1523-1533.

[23] M. Brahimi, K. Boukhalfa, and A. Moussaoui, "Deep learning for tomato diseases: classification and symptoms visualization," Applied Artificial Intelligence, vol. 31, no. 4, pp. 299-315, 2017, doi: 10.1080/08839514.2017.1315516.

[24] H. Durmuş, E. O. Güneş, and M. Kırc1, "Disease detection on the leaves of the tomato plants by using deep learning," 2017 6th International Conference on Agro-Geoinformatics, 2017, pp. 1-5, doi: 10.1109/Agro-Geoinformatics.2017.8047016.

[25] H. Hong, J. Lin, and F. Huang, "Tomato Disease Detection and Classification by Deep Learning," 2020 International Conference on Big Data, Artificial Intelligence and Internet of Things Engineering (ICBAIE), 2020, pp. 25-29, doi: 10.1109/ICBAIE49996.2020.00012.

[26] Y. Altuntas and A. F. Kocamaz, "Deep Feature Extraction for Detection of Tomato Plant Diseases and Pests based on Leaf Images," Celal Bayar University Journal of Science, vol. 17, no. 2, pp. 145-152, 2021, doi: 10.18466/cbayarfbe.812375. 


\section{BIOGRAPHIES OF AUTHORS}
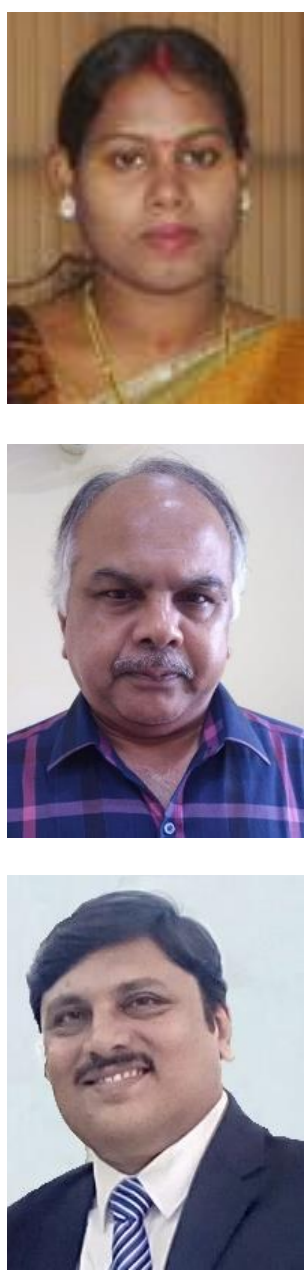

Rina Mahakud (D) SC $\mathrm{P}$ has completed her Bachelor in Electronics and Telecommunication Engg. and Master in Natural language processing from Utkal University, Odisha, India. Presently she is working as Assistant Professor in Dept of Electronics and Telecomm Engg. at Trident Academy of Technology, Bhubaneswar, Odisha, India. She has 13 years of teaching experience and published more than 7 research papers in journal and conferences. Her area of research interest is Machine learning and Internet of Things and guided numerous B. Tech Projects. She is a lifetime member of ISTE and IETE. She can be contacted at email: rinamahakud@gmail.com.

Dr. Binod Kumar Pattanayak (D) 81 SC P completed his M. S. in Computer Engineering from Kharkov Polytechnic Institute, Kharkov, Ukraine in the year 1992 and Ph. D. in Computer Science and Engineering from Siksha 'O' Anusandhan University, Bhubaneswar, Odisha, India in the year 2011. He has a teaching experience of 21 years in undergraduate, post graduate and Ph. D. Levels in computer science and engineering. He has successfully guided $\mathrm{Ph}$. D and $\mathrm{M}$. Tech. Level students. He is currently Professor in the department of computer science and engineering in Institute of Technical Education and Research (ITER), the faculty of engineering of S.O.A (Deemed to be) University, Bhubaneswar, Odisha, India. His research areas include ad hoc networks, software engineering, artificial intelligence and compiler design. He can be contacted at email: binodpattanayak@soa.ac.in.

Dr. Bibudhendu Pati D ID SC P is Associate Professor and Head in the Department of Computer Science at Rama Devi Women's University, Bhubaneswar, India. He has around 21 years of experience in teaching and research. His areas of research interests include Wireless Sensor Networks, Cloud Computing, Big Data, Internet of Things, and Advanced Network Technologies. He completed his Ph.D. from IIT Kharagpur, India. He is a Life Member of Indian Society for Technical Education, Computer Society of India and Senior Member of IEEE. He also served as Guest Editor of IJCNDS and IJCSE journals. He can be contacted at email: patibibudhendu@gmail.com. 